\title{
Modeling of a Circular Plate with Piezoelectric Actuators of Arbitrary Shape
}

\author{
M. WICIAK ${ }^{a, *}$ AND R. TROJANOWSKI ${ }^{b}$ \\ ${ }^{a}$ Cracow University of Technology, Institute of Mathematics, Warszawska St. 24, 31-155 Cracow, Poland \\ ${ }^{b} \mathrm{AGH}$ - University of Science and Technology, Faculty of Mechanical Engineering and Robotics \\ Department of Mechanics and Vibroacoustics, al. A. Mickiewicza 30, 30-059 Krakow, Poland
}

\begin{abstract}
This paper is concerned with mathematical aspects and numerical modeling of vibration of a circular plate with piezoelectric actuators. Particularly, a thin Kirchhoff-Love plate with arbitrary shaped actuators (e.g. pie-shaped, trapezoid, disc, and rectangular) is considered. In the theoretical model, the moments that act upon a structure and are induced by piezoelectric actuators are described by the generalized tensor product of a distribution and distribution-valued function. Numerical computations utilize the FEM approach supported by Ansys software.
\end{abstract}

DOI: $10.12693 /$ APhysPolA.123.1048

PACS: 43.40.At, 43.40.Vn

\section{Introduction}

The possibility of active vibration control for minimization of acoustic energy radiated by vibrating surface elements was explored for over 50 years [1]. The advantage of using differently shaped, distributed actuators for active control was demonstrated by a number of researchers, e.g. [2-5]. Many works deal with different problems like optimal placement of actuators $[6,7]$, using specific configurations [8]. There are also works that try to use elements typically used in active methods in passive systems $[9,10]$. Among plates of various shapes, circular plates seem to have a particular importance [5] due to their axial symmetry. Circular geometries are used in a wide variety of applications and are often easily manufactured. In [11], a thin rectangular plate with arbitrary shaped actuators (e.g. triangles, parallelograms, discs) is considered. The theoretical model for a structure is given in a language of distribution-valued function. Also, the formula for the solution of the Cauchy problem in the class of absolutely continuous tempered distribution-valued functions is derived.

This paper presents an analytical approach to modeling circular plates with piezoelectric actuators of arbitrary shape. In particular, pie-, trapezoid-, disc-, and rectangular-shaped actuators are considered. The natural tool to describe a structure that consists of a plate and a piezoelectric actuator is the theory of distributions. The moments that act upon a structure and are induced by piezoelectric actuators are expressed as the generalized tensor product of a distribution and distributionvalued function. For two actuator shapes (rectangular and disc), numerical models of a circular plate with two piezo elements attached are created. The analysis uses

*corresponding author; e-mail: mwiciak@pk.edu.pl the FEM method for structural vibrations. One of the piezo elements acts as an exciter and the other as an actuator for vibration reduction.

\section{Mathematical formulation of the problem}

\subsection{Equation of motion}

Let us consider a thin circular plate of thickness $h_{0}$ and radius $R$, and under the action of external forces and moments. The plate is also assumed to be made of linearly elastic, homogeneous and isotropic material of mass density $\rho$. The equation of motion for this plate is $([5])$ :

$$
D \nabla^{4} w+D \mu \frac{\partial}{\partial t} \nabla^{4} w+\rho h_{0} \frac{\partial^{2} w}{\partial t^{2}}=F,
$$

where $w=w(t, r, \theta)$ is transverse displacements, $\nabla^{4}=$ $\nabla^{2} \nabla^{2}$, and

$$
\nabla^{2}=\frac{\partial^{2}}{\partial r^{2}}+\frac{1}{r} \frac{\partial}{\partial r}+\frac{1}{r^{2}} \frac{\partial^{2}}{\partial \theta^{2}}
$$

is the Laplacian operator in polar coordinates $r, \theta$. Moreover, $F=F(t, r, \theta)$ is the external excitation, $\mu$ is internal damping loss factor, $D=E h_{0}^{3} / 12\left(1-\nu^{2}\right)$ is the flexural rigidity of the plate, $\nu$ and $E$ are the Poisson ratio and Young's modulus, respectively.

Additionally, consider a piezoelectric actuator adhered to the plate. The actuator consists of two identical pie-shaped piezoelectric elements of thickness $h$, bonded symmetrically on two opposite surfaces of the plate. The two elements are excited by opposite voltages and the actuator generates external excitation of the distributed moment type in the plate. It is assumed that the reaction moments $m_{r}$ and $m_{\theta}$ in polar coordinates are equal and uniformly distributed. Let $F_{p e}=F_{p e}(t, r, \theta)$ be an additional external stress caused by activating the piezoelectric element. Then $([1,5])$ :

$$
F_{p e}=\frac{\partial^{2} m_{r}}{\partial r^{2}}+\frac{2}{r} \frac{\partial m_{r}}{\partial r}+\frac{1}{r^{2}} \frac{\partial^{2} m_{\theta}}{\partial \theta^{2}}-\frac{1}{r} \frac{\partial m_{\theta}}{\partial r}
$$

and 


$$
m_{r}=m_{\theta}=m=C_{0} \epsilon_{p e} \chi,
$$

where $C_{0}$ denotes the piezoelectric strain-plate moment coupling term, $\epsilon_{p e}=d_{31} V / h$ is the magnitude of the induced strain, $d_{31}$ piezoelectric-strain constant, $V$ is the applied voltage, and $\chi$ is a characteristic function defined on the area of the piezoelectric actuator $\Gamma$,

$$
\chi(r, \theta)=\left\{\begin{array}{l}
1 \text { if }(r, \theta) \in \Gamma \\
0 \text { if }(r, \theta) \notin \Gamma
\end{array} .\right.
$$

The function $\chi$ is not differentiable (even not continuous) but the derivatives of $m_{r}=m_{\theta}=m$ can be understood in the sense of the theory of distribution. Namely, any locally summable function $\chi: \mathbb{R}^{2} \rightarrow \mathbb{R}$ defines regular distribution by means the formula

$$
[\chi](\varphi)=\int_{\mathbb{R}^{2}}^{2} \chi(r, \theta) \varphi(r, \theta) \mathrm{d} r \mathrm{~d} \theta
$$

for any test function $\varphi \in D\left(\mathbb{R}^{2}\right)$. Since $\chi$ is assumed to be a characteristic function on $\Gamma$

$$
[\chi](\varphi)=\int_{\Gamma} \varphi(r, \theta) \mathrm{d} r \mathrm{~d} \theta
$$

for $\varphi \in D\left(\mathbb{R}^{2}\right)$. Let us also recall that for any multi-index $\alpha \in \mathbb{N}^{2}$, the derivative $D^{\alpha} T$ of distribution $T \in D^{\prime}\left(\mathbb{R}^{2}\right)$ is given by

$$
D^{\alpha} T(\varphi)=(-1)^{|\alpha|} T\left(\frac{\partial^{|\alpha|}}{\partial r^{\alpha_{1}} \partial \theta^{\alpha_{2}}} \varphi\right)
$$

for $\varphi \in D\left(\mathbb{R}^{2}\right)$, where $\alpha=\left(\alpha_{1}, \alpha_{2}\right)$ and $|\alpha|=\alpha_{1}+\alpha_{2}$. Thus one can see that the formulae for distributions $\frac{\partial^{2} m}{\partial r^{2}}$, $\frac{\partial m}{\partial r}, \frac{\partial^{2} m}{\partial \theta^{2}}$ and $\frac{\partial m}{\partial r}$ in (3) depend on the shape of $\Gamma$.

\subsection{Pie-shaped actuator}

Consider at first a pie-shaped actuator bonded to the plate as shown in Fig.1. In this case

$$
\Gamma=\left\{(r, \theta): R_{1} \leq r \leq R_{2}, \theta_{1} \leq \theta \leq \theta_{2}\right\} .
$$

It is convenient $[1,2,4,11]$ to treat the distribution $[\chi]$ as a tensor product,

$$
[\chi]=\left[H_{\theta_{1}}-H_{\theta_{2}}\right] \otimes\left[H_{R_{1}}-H_{R_{2}}\right],
$$

where $H_{a}$ is a Heaviside step function, $H_{a}(x)=1$ for $x \geq a$ and $H_{a}(x)=0$ for $x<a$.

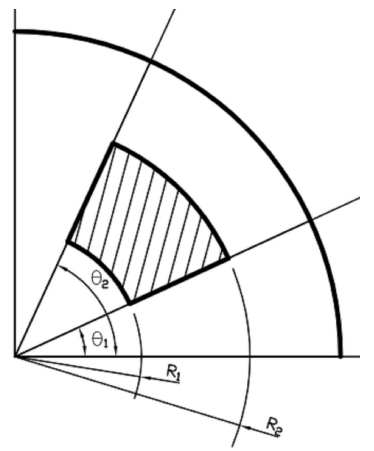

Fig. 1. Pie-shaped piezo actuator.

Let us recall $[12,11]$ that tensor product of distributions $T, S \in D^{\prime}(\mathbb{R})$ is a distribution $S \otimes T \in D^{\prime}\left(\mathbb{R}^{2}\right)$ defined as

$$
(S \otimes T)(\varphi)=S(\theta \longmapsto T(\varphi(\cdot, \theta)) .
$$

In the above, the distribution $T \in D^{\prime}(\mathbb{R})$ operates on test functions of variable $r$, while $S \in D^{\prime}(\mathbb{R})$ is a distribution on test functions of variable $\theta$. It is well known that for any $\varphi \in D\left(\mathbb{R}^{2}\right)$, the mappings $\mathbb{R} \ni r \mapsto \varphi(r, \theta) \in \mathbb{R}$ for fixed $\theta$, and $\mathbb{R} \ni \theta \mapsto T(\varphi(\bullet, \theta)) \in \mathbb{R}$ are test functions of variables $r$ and $\theta$, respectively. It is also known [12] that the following formulae for derivatives are true:

$$
\begin{aligned}
& \frac{\partial^{k}}{\partial r^{k}}(S \otimes T)=S \otimes \frac{\mathrm{d}^{k}}{\mathrm{~d} r^{k}} T, \frac{\partial^{k}}{\partial \theta^{k}}(S \otimes T) \\
& \quad=\frac{\mathrm{d}^{k}}{\mathrm{~d} \theta^{k}} S \otimes T .
\end{aligned}
$$

According to (8) one can easy compute

$$
\begin{aligned}
& \frac{\mathrm{d}}{\mathrm{d} x}\left[H_{a}\right](\varphi)=-\left[H_{a}\right]\left(\varphi^{\prime}\right)=-\int_{a}^{\infty} \varphi^{\prime}(x) \mathrm{d} x= \\
& \varphi(a)=\delta_{a}(\varphi)
\end{aligned}
$$

for $\varphi \in D(\mathbb{R})$, where $\delta_{a}$ is the Dirac distribution and for any $\varphi \in D(\mathbb{R}), \delta_{a}(\varphi)=\varphi(a)$. Thus

$$
\frac{\mathrm{d}}{\mathrm{d} x}\left[H_{a}\right]=\delta_{a} \text {. }
$$

Thereby on account of (4), (10), (12), (14) one can obtain

$$
\begin{aligned}
& \frac{\partial m}{\partial r}=C_{0} \epsilon_{p e}\left[H_{\theta_{1}}-H_{\theta_{2}}\right] \otimes\left(\delta_{R_{1}}-\delta_{R_{2}}\right), \\
& \frac{\partial^{2} m}{\partial r^{2}}=C_{0} \epsilon_{p e}\left[H_{\theta_{1}}-H_{\theta_{2}}\right] \otimes\left(\delta_{R_{1}}^{\prime}-\delta_{R_{2}}^{\prime}\right), \\
& \frac{\partial^{2} m}{\partial \theta^{2}}=C_{0} \epsilon_{p e}\left(\delta_{\theta_{1}}^{\prime}-\delta_{\theta_{2}}^{\prime}\right) \otimes\left[H_{R_{1}}-H_{R_{2}}\right],
\end{aligned}
$$

where, for short, $\delta_{R_{i}}^{\prime}, \delta_{\theta_{i}}^{\prime}$ denote $\frac{\mathrm{d}}{\mathrm{d} r} \delta_{R_{i}}$ and $\frac{\mathrm{d}}{\mathrm{d} \theta} \delta_{\theta_{i}}$, derivatives of distributions $\delta_{R_{i}}, \delta_{\theta_{i}} \in D(\mathbb{R})$, respectively $(i=1,2)$. Finally, the external loads due to the actuator are

$$
\begin{aligned}
& F_{p e}=C_{0} \epsilon_{p e}\left(\left[H_{\theta_{1}}-H_{\theta_{2}}\right]\right. \\
& \otimes\left(\delta_{R_{1}}^{\prime}-\delta_{R_{2}}^{\prime}+\frac{1}{r} \delta_{R_{1}}-\frac{1}{r} \delta_{R_{2}}\right) \\
& \left.+\frac{1}{r^{2}}\left(\delta_{\theta_{1}}^{\prime}-\delta_{\theta_{2}}^{\prime}\right) \otimes\left[H_{R_{1}}-H_{R_{2}}\right]\right) .
\end{aligned}
$$

\subsection{Trapezoid or disk-shaped actuator}

Now, let us turn to the case of a bit more complicated shapes of piezoelectric actuators. Consider trapezoid or circular actuator which is bonded to the plate as shown in Fig. 2 and Fig. 3, respectively. It is assumed that parallel sides of a trapezoid-shaped actuator are also parallel to a tangent to the plate edge, and are given by the equations $y=a x+b_{1}, y=a x+b_{2}, 0<b_{1}<b_{2}<R$. As far as a disk-shaped actuator is considered it is claimed that the actuator is enclosed by a circle with the centre at the point $(a, b)$ and a radius $r_{0}$. Analogously to the case of pie-shaped actuator the internal moments across the piezoelectric can be expressed as (4) and (5), where

$$
\Gamma=\left\{(r, \theta): r_{1}(\theta) \leq r \leq r_{2}(\theta), \theta_{1} \leq \theta \leq \theta_{2}\right\},
$$




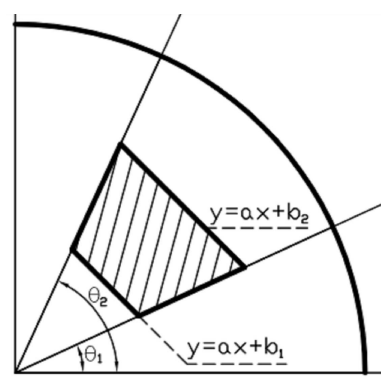

Fig. 2. Trapezoid shaped piezo actuator.

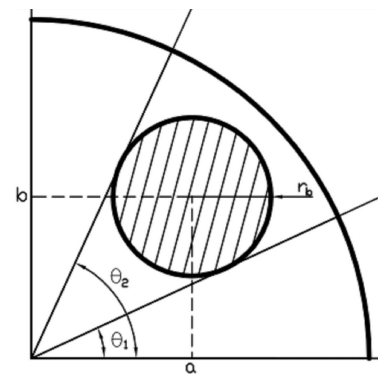

Fig. 3. Disc-shaped piezo actuator.

$$
r_{i}(\theta)=\frac{b_{i}}{\sin \theta-a \cos \theta} \text { for } i=1,2,
$$

in the case of trapezoid-shaped actuator, and

$$
\begin{aligned}
& r_{1,2}(\theta)=a \cos \theta+b \sin \theta \\
& \mp \sqrt{r_{0}^{2}-(a \sin \theta-b \cos \theta)^{2}},
\end{aligned}
$$

in the case of disk-shaped actuator, where $\theta_{1}$ and $\theta_{2}$ are the slopes of the tangent to the circle from the center of the plate [5].

Unfortunately, in both above cases the distribution $[\chi]$ cannot be treated as a tensor product of distributions since $r_{i}$ is a function of $\theta$ and $\left[H_{r_{i}}\right]$ is not longer a distribution but a distribution-valued function, $\mathbb{R} \ni$ $\theta \mapsto\left[H_{r_{i}(\theta)}\right] \in D^{\prime}(\mathbb{R})$. A simple example showing that even when a distribution $S$ depends only on $\theta$ and $T(\theta) \in D^{\prime}(\mathbb{R})$ is a distribution depending on $r$, the tensor product is not a distribution, can be constructed [11]. For this reason, a generalized tensor product will be used.

For the convenience of the reader, we recall that if $S \in D^{\prime}(\mathbb{R})$ and a distribution-valued function $T: \mathbb{R} \ni$ $\theta \mapsto T(\theta) \in D^{\prime}(\mathbb{R})$ is of class $C^{\infty}$, then the generalized tensor product of $S$ and $T, S \otimes T$, is a distribution, and is defined by

$$
(S \otimes T) \varphi=S(\theta \mapsto T(\theta) \varphi(\cdot, \theta))
$$

for any $\varphi \in D\left(\mathbb{R}^{2}\right)$. The following formulae for derivatives of $S \otimes T$ are also true [11]:

$$
\frac{\partial^{k}}{\partial r^{k}}(S \otimes T)=S \otimes \frac{\mathrm{d}^{k}}{\mathrm{~d} r^{k}} T(\bullet),
$$

$$
\frac{\partial^{k}}{\partial \theta^{k}}(S \otimes T)=\sum_{i=0}^{k}\left(\begin{array}{l}
k \\
i
\end{array}\right) \frac{\mathrm{d}^{i}}{\mathrm{~d} \theta^{i}} S \otimes T^{(k-i)},
$$

where the symbols $\frac{\partial^{k}}{\partial r^{k}}, \frac{\mathrm{d}^{k}}{\mathrm{~d} r^{k}}, \frac{\partial^{k}}{\partial \theta^{k}}, \frac{\mathrm{d}^{i}}{\mathrm{~d} \theta^{i}}$ denote derivatives of distributions in accordance with $(8)$, while $T^{(j)}$ means the $j$-th derivative of the map $T$.

Consequently, the internal moments across the trapezoid- and disk-shaped piezoelectric element can be expressed as the generalized tensor product

$$
m_{r}=m_{\theta}=m=C_{0} \epsilon_{p e}\left[H_{\theta_{1}}-H_{\theta_{2}}\right]
$$

$$
\otimes\left[H_{r_{1}(\theta)}-H_{r_{2}(\theta)}\right] \text {. }
$$

Since for any $\theta \in \mathbb{R}$ and $\varphi \in D(\mathbb{R})$,

$$
\left[H_{r_{i}(\theta)}\right] \varphi=\int_{r_{i}(\theta)}^{\infty} \varphi(r) \mathrm{d} r, i=1,2,
$$

the derivative of the map $\mathbb{R} \ni \theta \mapsto\left[H_{r_{i}(\theta)}\right] \in D^{\prime}(\mathbb{R})$ is given by

$$
\left[H_{r_{i}(\theta)}\right]^{\prime}=-r_{i}^{\prime}(\theta) \cdot \delta_{r_{i}(\theta)} \text { for } i=1,2,
$$

and

$$
\left[H_{r_{i}(\theta)}\right]^{\prime \prime}=\left(r_{1}^{\prime}(\theta)\right)^{2} \cdot \delta_{r_{i}(\theta)}^{\prime}-r_{i}^{\prime \prime}(\theta) \cdot \delta_{r_{i}(\theta)},
$$

$$
\text { for } i=1,2 \text {. }
$$

Therefore from $(25,24)$ the second derivative of $m$ with respect to $\theta$ is obtained as equaling

$$
\begin{aligned}
& \frac{\partial^{2} m}{\partial \theta^{2}}=C_{0} \epsilon_{p e}\left([ H _ { \theta _ { 1 } } - H _ { \theta _ { 2 } } ] \otimes \left[\left(r_{1}^{\prime}(\theta)\right)^{2} \bullet \delta_{r_{1}(\theta)}^{\prime}\right.\right. \\
& \left.\quad-\left(r_{2}^{\prime}(\theta)\right)^{2} \bullet \delta_{r_{2}(\theta)}^{\prime}+r_{1}^{\prime \prime}(\theta) \cdot \delta_{r_{1}(\theta)}-r_{2}^{\prime \prime}(\theta) \bullet \delta_{r_{2}(\theta)}\right] \\
& \quad-2\left(\delta_{\theta_{1}}-\delta_{\theta_{2}}\right) \otimes\left(r_{1}^{\prime}(\theta) \bullet \delta_{r_{1}(\theta)}-r_{2}^{\prime}(\theta) \cdot \delta_{r_{2}(\theta)}\right) \\
& \left.\quad+\left(\delta_{\theta_{1}}^{\prime}-\delta_{\theta_{2}}^{\prime}\right) \otimes\left[H_{r_{1}(\theta)}-H_{r_{2}(\theta)}\right]\right) .
\end{aligned}
$$

Similarly, in accordance with $(25,23)$, the derivatives of $m$ with respect to $r$ are

$$
\begin{gathered}
\frac{\partial m}{\partial r}=C_{0} \epsilon_{p e}\left(\left[H_{\theta_{1}}-H_{\theta_{2}}\right] \otimes \frac{\mathrm{d}}{\mathrm{d} r}\left[H_{r_{1}(\theta)}-H_{r_{2}(\theta)}\right]\right) \\
=C_{0} \epsilon_{p e}\left(\left[H_{\theta_{1}}-H_{\theta_{2}}\right] \otimes\left(\delta_{r_{1}(\theta)}-\delta_{r_{2}(\theta)}\right)\right),
\end{gathered}
$$

and

$$
\frac{\partial^{2} m}{\partial \mathbb{R}^{2}}=C_{0} \epsilon_{p e}\left(\left[H_{\theta_{1}}-H_{\theta_{2}}\right] \otimes\left(\delta_{r_{1}(\theta)}^{\prime}-\delta_{r_{2}(\theta)}^{\prime}\right)\right) .
$$

Consequently,

$$
\begin{aligned}
& F_{p e}=\left[H_{\theta_{1}}-H_{\theta_{2}}\right] \otimes\left(\left(1+\frac{1}{r^{2}}\left(r_{1}^{\prime}(\theta)\right)^{2}\right) \delta_{r_{1}(\theta)}^{\prime}\right. \\
& -\left(1+\frac{1}{r^{2}}\left(r_{2}^{\prime}(\theta)\right)^{2}\right) \delta_{r_{2}(\theta)}^{\prime} \\
& \quad+\frac{1}{r^{2}}\left(\left(r+r_{1}^{\prime \prime}(\theta)\right) \delta_{r_{1}(\theta)}-\left(r+r_{2}^{\prime \prime}(\theta)\right) \delta_{r_{2}(\theta)}\right) \\
& +\frac{1}{r^{2}}\left(\delta_{\theta_{1}}^{\prime}-\delta_{\theta_{2}}^{\prime}\right) \otimes\left[H_{r_{1}(\theta)}-H_{r_{2}(\theta)}\right] \\
& \left.\quad-2 \frac{1}{r^{2}}\left(\delta_{\theta_{1}}-\delta_{\theta_{2}}\right) \otimes\left(r_{1}^{\prime}(\theta) \bullet \delta_{r_{1}(\theta)}-r_{2}^{\prime}(\theta) \bullet \delta_{r_{2}(\theta)}\right)\right) .
\end{aligned}
$$

In particular, in the case of trapezoid-shaped actuator, 


$$
r_{i}^{\prime}(\theta)=-\frac{b_{i}}{(\sin \theta-a \cos \theta)^{2}}(\cos \theta+a \sin \theta),
$$

and

$$
\begin{aligned}
& r_{i}^{\prime \prime}(\theta)=\frac{b_{i}}{(\sin \theta-a \cos \theta)^{3}} \\
& \quad \times\left(2+a^{2}-\left(1-a^{2}\right) \sin ^{2} \theta+a \sin (2 \theta)\right),
\end{aligned}
$$

whereas, in the case of disk-shaped actuator, derivatives of $r_{i}$ are given by

$$
\begin{aligned}
& r_{1,2}^{\prime}(\theta)=-a \sin \theta+b \cos \theta \\
& \quad \pm \frac{\left(a^{2}-b^{2}\right) \sin 2 \theta-2 a b \cos 2 \theta}{2 \sqrt{r_{0}^{2}-(a \sin \theta-b \cos \theta)^{2}}},
\end{aligned}
$$

and so

$$
\begin{aligned}
& r_{1,2}^{\prime \prime}(\theta)=-a \cos \theta-b \sin \theta \\
& \pm \frac{\left(a^{2}-b^{2}\right) \cos 2 \theta+a b \sin 2 \theta^{2}}{r_{0}^{2}-(a \sin \theta-b \cos \theta)} \\
& \mp \frac{1}{8} \frac{\left(\left(a^{2}-b^{2}\right) \sin 2 \theta-2 a b \cos 2 \theta\right)^{2}}{r_{0}^{2}-(a \sin \theta-b \cos \theta)^{2}} .
\end{aligned}
$$

\subsection{Rectangular actuators}

Consider now a rectangular actuator which is bonded to the plate as shown in Fig. 4. Let us assume that two parallel sides of the rectangle are parallel to a tangent to the plate edge, and are represented by the equations $y=a x+b_{1}, y=a x+b_{2}, 0<b_{1}<b_{2}<R$. Let $y=-1 / a x+b_{i}, i=3,4, b_{3}<b_{4}$, denote the equations of two other parallel sides of rectangle.

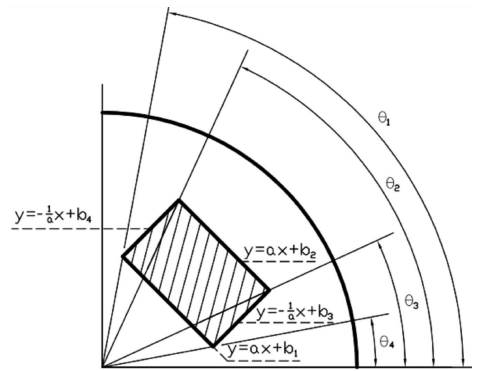

Fig. 4. Rectangular shaped piezo actuator.

Denote also by $\left(x_{i}, y_{i}\right)$ the vertexes of the rectangle for $i=1, \ldots, 4$ and $\theta_{i}=\tan ^{-1}\left(y_{i} / x_{i}\right)$, as shown in Fig. 4 . Analogously to previous sections, the reaction moments across the rectangular actuator can be described by $m_{r}=$ $m_{\theta}=m=C_{0} \epsilon_{p e}[\chi]$, where $\chi$ is a characteristic function of the region $\Gamma$ covered by the rectangular actuator [3].

To provide the parameterization of $\Gamma$ in polar coordinates we need to decompose it into three subregions, $\Gamma=\Gamma_{1} \cup \Gamma_{2} \cup \Gamma_{3}$, determined by $\Gamma_{1}=\left\{(r, \theta): r_{1}(\theta) \leq r \leq\right.$ $\left.r_{4}(\theta), \theta_{2} \leq \theta \leq \theta_{1}\right\}, \Gamma_{2}=\left\{(r, \theta): r_{1}(\theta) \leq r \leq r_{2}(\theta), \theta_{4} \leq\right.$ $\left.\theta \leq \theta_{2}\right\}$, and $\Gamma_{3}=\left\{(r, \theta): r_{1}(\theta) \leq r \leq r_{3}(\theta), \theta_{3} \leq \theta \leq\right.$ $\left.\theta_{4}\right\}$, where

$$
r_{i}(\theta)=\frac{b_{i}}{\sin \theta-a \cos \theta} \text { for } i=1,2,
$$

$$
r_{i}(\theta)=\frac{b_{i}}{\sin \theta+\frac{1}{a} \cos \theta} \text { for } i=3,4 .
$$

Thus the distribution $[\chi]$ can be expressed as a sum

$$
\begin{gathered}
{[\chi]=\left[H_{\theta_{2}}-H_{\theta_{1}}\right] \otimes\left[H_{r_{1}(\theta)}-H_{r_{4}(\theta)}\right]} \\
+\left[H_{\theta_{4}}-H_{\theta_{2}}\right] \otimes\left[H_{r_{1}(\theta)}-H_{r_{2}(\theta)}\right] \\
+\left[H_{\theta_{3}}-H_{\theta_{4}}\right] \otimes\left[H_{r_{1}(\theta)}-H_{r_{3}(\theta)}\right] .
\end{gathered}
$$

With (3) taken into account an easy computation shows that

$$
\begin{aligned}
& F_{p e}=C_{0} \epsilon_{p e} \\
& \quad \times\left([ H _ { \theta _ { 3 } } - H _ { \theta _ { 1 } } ] \otimes \frac { 1 } { r ^ { 2 } } \left[\left(r^{2}+\left(r_{1}^{\prime}(\theta)\right)^{2}\right) \delta_{r_{1}(\theta)}^{\prime}\right.\right. \\
& \left.\quad-\left(r+r_{1}^{\prime \prime}(\theta)\right) \delta_{r_{1}(\theta)}\right]+\left[H_{\theta_{2}}-H_{\theta_{4}}\right] \\
& \quad \otimes \frac{1}{r^{2}}\left[\left(r^{2}+\left(r_{2}^{\prime}(\theta)\right)^{2}\right) \delta_{r_{2}(\theta)}^{\prime}-\left(r+r_{2}^{\prime \prime}(\theta)\right) \delta_{r_{2}(\theta)}\right] \\
& +\left[H_{\theta_{4}}-H_{\theta_{3}}\right] \otimes \frac{1}{r^{2}}\left[\left(r^{2}+\left(r_{3}^{\prime}(\theta)\right)^{2}\right) \delta_{r_{3}(\theta)}^{\prime}\right. \\
& \left.-\left(r+r_{3}^{\prime \prime}(\theta)\right) \delta_{r_{3}(\theta)}\right]+\left[H_{\theta_{1}}-H_{\theta_{2}}\right] \\
& \otimes \frac{1}{r^{2}}\left[\left(r^{2}+\left(r_{4}^{\prime}(\theta)\right)^{2}\right) \delta_{r_{4}(\theta)}^{\prime}-\left(r+r_{4}^{\prime \prime}(\theta)\right) \delta_{r_{4}(\theta)}\right] \\
& \quad \frac{1}{r^{2}}\left[\delta_{\theta_{1}}^{\prime} \otimes\left[H_{r_{4}}-H_{r_{1}}\right]+\delta_{\theta_{2}}^{\prime} \otimes\left[H_{r_{2}}-H_{r_{4}}\right]\right. \\
& \left.+\delta_{\theta_{3}}^{\prime} \otimes\left[H_{r_{1}}-H_{r_{3}}\right]+\delta_{\theta_{4}}^{\prime} \otimes\left[H_{r_{3}}-H_{r_{2}}\right]\right] \\
& +\frac{2}{r^{2}} \delta_{\theta_{1}} \otimes\left[\left(r_{1}^{\prime} \delta_{r_{1}}-r_{4}^{\prime} \delta_{r_{4}}\right)+\delta_{\theta_{2}} \otimes\left(r_{4}^{\prime} \delta_{r_{4}}-r_{2}^{\prime} \delta_{r_{2}}\right)\right. \\
& \left.\left.+\delta_{\theta_{3}} \otimes\left(r_{3}^{\prime} \delta_{r_{3}}-r_{1}^{\prime} \delta_{r_{1}}\right)+\delta_{\theta_{4}} \otimes\left(r_{2}^{\prime} \delta_{r_{2}}-r_{3}^{\prime} \delta_{r_{3}}\right)\right]\right)
\end{aligned}
$$

Note that derivatives of curves $r_{i}=r_{i}(\theta)$ for $i=1,2$ are given by formulae (33), (34), while for $i=3,4$ one can obtain

$$
r_{i}^{\prime}=-\frac{b_{i}}{\left(\sin \theta+\frac{1}{a} \cos \theta\right)^{2}}\left(\cos \theta-\frac{1}{a} \sin \theta\right),
$$

and

$$
\begin{aligned}
r_{i}^{\prime \prime} & =\frac{b_{i}}{\left(\sin \theta+\frac{1}{a} \cos \theta\right)^{3}} \\
& \times\left(2+\frac{1}{a^{2}}-\left(1-\frac{1}{a^{2}}\right) \sin ^{2} \theta-\frac{1}{a} \sin 2 \theta\right) .
\end{aligned}
$$

Finally it should be noted that it is possible to describe external loads due to another shapes of actuators in analogous way. Also one can build a model for transverse displacement when a set of actuators is considered. In this case the term of external loads is a sum of distributions that describe single actuator.

Let us note that the solution of (1) exists in the sense of the theory of distributions. The theorem on existence 
of the solution and analytic formulae for the solution can be found in [13]. Equation (1) can be also solved using classical methods based on the separation of variables $[2,3]$. An approximate solution can be obtained by the finite element method.

\section{Numerical calculations}

From the above analytical considerations two actuator shapes were chosen for numerical simulations. Those were disc where the added external is describe by equations (32), (35), (36) and rectangle where the added external is described by equations (39)-(41), (33), and (34). Four models consisting of a steel plate with a diameter equal to $0.15 \mathrm{~m}$ and thickness equal to $0.001 \mathrm{~m}$ clamped on the edge and two piezo elements attached were created. The first element marked by white color is used for plate excitation but the other one marked by violet color for vibration reduction. In the first two models the primary excitation was obtained with the use of square actuator and for reduction a square one and a disc-shaped one were used (Fig. 5a and b). For the next models the difference was that the piezo element used for plates excitation were disc-shaped (Fig. 5c and d).
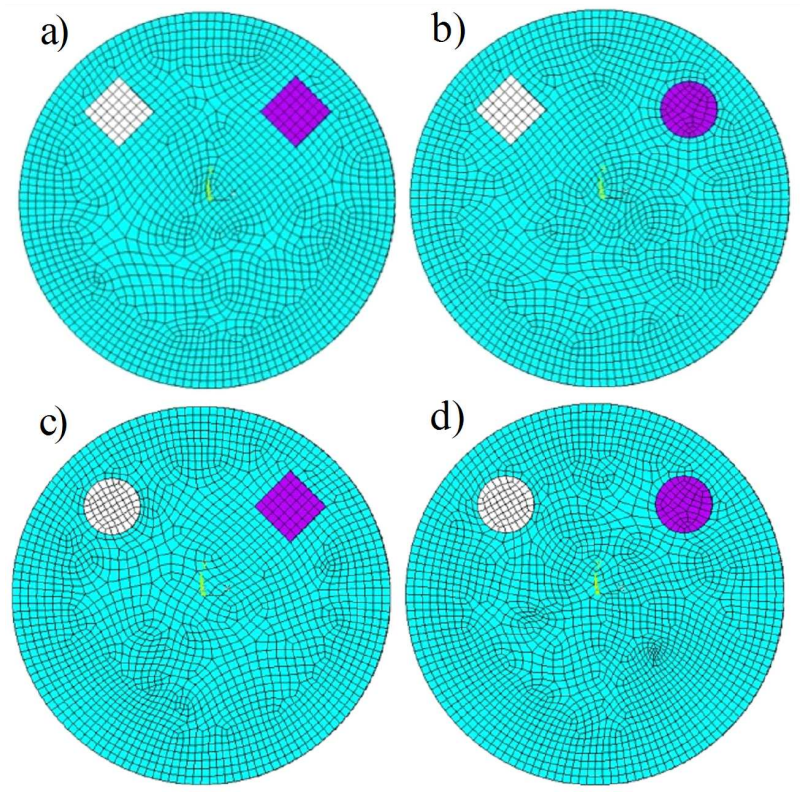

Fig. 5. Created models.

All of the modeled piezo actuators had a base area of $1600 \mathrm{~m}^{2}$ and $1 \mathrm{~mm}$ thickness. The element used for modeling were SOLSH190 for the plate and SOLID226 for actuators. Material constants can be found in Table I.

After performing modal analyses, 6 first modes were chosen for harmonic analyses (although mode 2 and 3 occur for almost the same frequency, so in harmonic analyses they were treated as one). Fig. 6 shows those mode shapes.

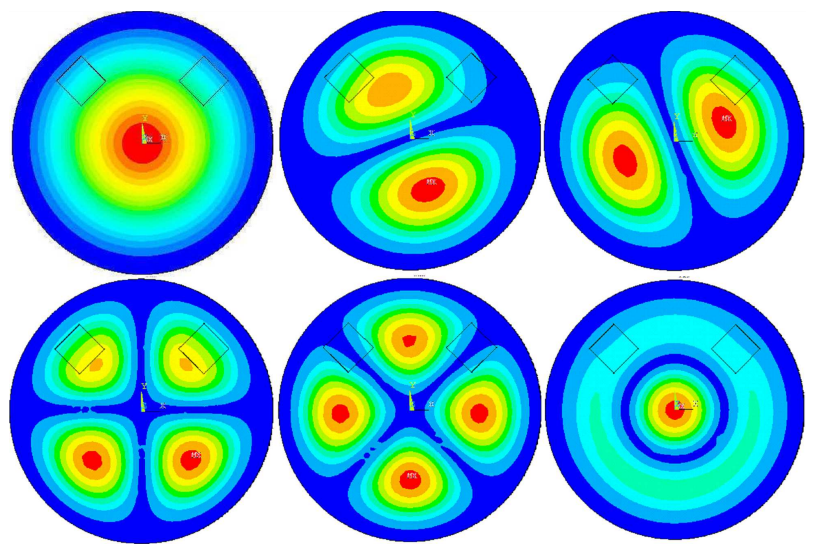

Fig. 6. Modes shapes analyzed.

TABLE I

Model parameters.

\begin{tabular}{l|c|l}
\hline \hline \multicolumn{1}{c|}{ Object } & Element type & \multicolumn{1}{c}{ Other info } \\
\hline plate & SOLSH190 & $\rho=7500 \mathrm{~kg} / \mathrm{m}^{3}$, \\
& & $\begin{array}{l}E=2.1 \times 10^{11} \mathrm{~Pa}, \\
\nu=0.3\end{array}$ \\
\hline piezo element & SOLID226 & $\begin{array}{l}\text { equivalent to PZT4, } \\
A=600 \mathrm{~mm}^{2}\end{array}$
\end{tabular}

For frequencies corresponding to the chosen mode shapes, harmonic analyses were performed with optimization procedure for the voltage applied to the element used for vibration reduction. The goal function for optimization was

$$
J=\min \left(\frac{\sum_{i=1}^{n} u_{z i}}{n}\right),
$$

where $u_{z i}$ are vibrations of node $i$ of the plate in the plane perpendicular to its base, and $n$ number of nodes creating plates base.

Optimization parameters are shown in Table II, where $V_{0}$ is the voltage amplitude applied to the element used for plates excitation while $V_{1}$ and $\varphi_{1}$ are design variables. $V_{1}$ is the voltage amplitude applied to the actuator and $\varphi_{1}$ is a phase angle of applied voltage as described by the equation

$$
V=V_{1} e^{j\left(\omega t+\varphi_{1}\right)}
$$

where $j^{2}=-1$. The maximum number of iterations was set to 200 .

TABLE II

Optimization parameters.

\begin{tabular}{c|c}
\hline \hline Quantity & Range \\
\hline$V_{0}$ & $100 \mathrm{~V}$ \\
$V_{1}$ & $0.1-200 \mathrm{~V}$ \\
$\varphi_{1}$ & $0-360^{\circ}$
\end{tabular}




\section{Results and conclusions}

Obtained results are presented in Table III.

TABLE III

Results of the analyses.

\begin{tabular}{|c|c|c|c|c|c|}
\hline \multirow[t]{2}{*}{ mode } & \multicolumn{2}{|c|}{ shape } & \multirow{2}{*}{$\begin{array}{r}V_{1} \\
{[\mathrm{~V}]}\end{array}$} & \multirow{2}{*}{$\begin{array}{c}\phi_{1} \\
{[\mathrm{deg}]}\end{array}$} & \multirow{2}{*}{$\begin{array}{c}\text { reduction } \\
{[\mathrm{dB}]}\end{array}$} \\
\hline & excitation & reduction & & & \\
\hline \multirow{4}{*}{1} & \multirow{2}{*}{ square } & square & 100.30 & 180.00 & 34.52 \\
\hline & & disc & 104.56 & 179.14 & 30.64 \\
\hline & \multirow{2}{*}{$\operatorname{disc}$} & square & 98.41 & 179.80 & 32.97 \\
\hline & & disc & 100.56 & 179.78 & 33.97 \\
\hline \multirow{4}{*}{2 or 3} & \multirow{2}{*}{ square } & square & 17.44 & 50.45 & 0.18 \\
\hline & & disc & 17.44 & 50.45 & 0.17 \\
\hline & \multirow{2}{*}{ disc } & square & 19.76 & 62.72 & 0.18 \\
\hline & & disc & 15.28 & 26.95 & 0.15 \\
\hline \multirow{4}{*}{4} & \multirow{2}{*}{ square } & square & 100.35 & 0.00 & 38.83 \\
\hline & & disc & 105.68 & 359.39 & 36.73 \\
\hline & \multirow{2}{*}{ disc } & square & 94.92 & 0.04 & 37.29 \\
\hline & & disc & 100.38 & 0.47 & 37.32 \\
\hline \multirow{4}{*}{5} & \multirow{2}{*}{ square } & square & 106.94 & 180.00 & 3.23 \\
\hline & & disc & 102.06 & 177.97 & 3.45 \\
\hline & \multirow{2}{*}{$\operatorname{disc}$} & square & 80.00 & 188.31 & 3.22 \\
\hline & & disc & 76.17 & 180.13 & 3.75 \\
\hline \multirow{4}{*}{6} & \multirow{2}{*}{ square } & square & 99.14 & 180.00 & 34.10 \\
\hline & & disc & 104.69 & 179.99 & 33.44 \\
\hline & \multirow{2}{*}{ disc } & square & 93.63 & 180.27 & 33.26 \\
\hline & & disc & 99.46 & 179.97 & 33.42 \\
\hline
\end{tabular}

Examining the results one can say that the differences in vibration reduction obtained with the use of different shapes of piezo actuators are rather small (with one exception), and depend on the relation of shapes between the element used for excitation and the one used for reduction. Another thing is that the largest reduction occurs for modes $(1,0),(1,2,0)$ and $(2,0)$, that occurs not only when our configuration is symmetrical but also when there is a proper placement of actuators.

The difference in results for disc-disc configuration for modes 2 or 3 is probably the effect of the second mode shape from this pair being observed.

It also appears that for most cases the square shaped elements need lower voltage to achieve similar effect (although the difference is rather small and it does not exceed $6 \%$ ).

In the paper an analytical model of circular plates with piezoelectric actuators of arbitrary shape was presented.
The internal moments across the actuator were described in terms of generalized tensor product of a distribution and a distribution-valued function. The solution of the problem exists in the sense of the theory of distributions. It can be derived by analytic formulae or using classical methods based on separation of variables. On the other hand an approximate solution can be obtained by the finite element method. Numerical analyses results showed large vibration reduction (more than $30 \mathrm{~dB}$ ) for the first, fourth and sixth mode, and almost no reduction for the rest of the analyzed modes. If the actuator was placed symmetrically to the exciter, all 6 modes could easily be reduced.

\section{Acknowledgments}

This study is a part of the research project $\mathrm{N}$ N504078038 supported by Polish Ministry of Science and Higher Education.

\section{References}

[1] E.K. Dimitriadis, C.R. Fuller, C.A. Rogers, $A I A A$ Journal 29, 1771 (1991).

[2] E.K. Dimitriadis, C.R. Fuller, C.A. Rogers, J. Vib. Acoust. 113, 100 (1991).

[3] E.M. Sekouri, Y. Hu, A.D. Ngo, Mechatronics 14 1007 (2004).

[4] M. Sullivan, J.E. Hubbard, Jr., S.E. Burke, J. Acoust. Soc. Amer. 99, 2965 (1996).

[5] J. Wiciak, Archives of Acoustics 32, 265 (2007).

[6] A. Brański, M. Borkowski, S. Szela, Acta Phys. Pol. A 118, 17 (2010).

[7] A. Brański, G. Lipiński, Acta Phys. Pol. A 119, 936 (2011).

[8] M. S. Kozień, J. Wiciak, Acta Phys. Pol. A 116, 348 (2009).

[9] R. Filipek, J. Wiciak, European Physical Journal Special Topics 154, 57 (2008).

[10] M.S. Kozień, B. Koltowski, Acta Phys. Pol. A 119, 1005 (2011).

[11] M. Wiciak, Acta Phys. Polon. A 121, A-142 (2012).

[12] W. Rudin, Functional Analysis, McGraw-Hill, New York, 1973.

[13] M. Wiciak, Univ. Iagel. Acta Math. 42, 31 (2004). 